\title{
The Use of Non-invasive Vagus Nerve Stimulation to Treat Respiratory Symptoms Associated With COVID-19: A Theoretical Hypothesis and Early Clinical Experience
}

\section{Peter Staats, MD*; Georgios Giannakopoulos, $\mathrm{DO}^{\dagger}$; Justyna Blake*; Eric Liebler* ${ }^{\circ}$; Robert M. Levy, MD, PhD ${ }^{\ddagger}$}

\begin{abstract}
Objectives: Coronavirus disease 2019 (COVID-19), caused by severe acute respiratory syndrome coronavirus 2 (SARS-CoV-2), is a pandemic with no specific therapeutic agents and substantial mortality, and finding new treatments is critical. Most cases are mild, but a significant minority of patients develop moderate to severe respiratory symptoms, with the most severe cases requiring intensive care and/or ventilator support. This respiratory compromise appears to be due to a hyperimmune reaction, often called a cytokine storm. Vagus nerve stimulation has been demonstrated to block production of cytokines in sepsis and other medical conditions. We hypothesize that non-invasive vagus nerve stimulation (nVNS) might provide clinical benefits in patients with respiratory symptoms similar to those associated with COVID-19.

Materials and Methods: Information on two case reports was obtained via email correspondence and phone interviews with the patients.

Results: Both patients reported clinically meaningful benefits from nVNS therapy. In case 1, the patient used nVNS to expedite symptomatic recovery at home after hospital discharge and was able to discontinue use of opioid and cough suppressant medications. In case 2, the patient experienced immediate and consistent relief from symptoms of chest tightness and shortness of breath, as well as an improved ability to clear his lungs.

Conclusions: Preliminary observations and a strong scientific foundation suggest that nVNS might provide clinical benefits in patients with COVID-19 via multiple mechanisms.

Keywords: Cholinergic anti-inflammatory pathway, COVID-19, cytokine storm, neuromodulation, non-invasive vagus nerve stimulation, respiratory symptoms

Conflict of Interest: Peter Staats, is an employee and was cofounder of electroCore, Inc., and receives stock ownership. Justyna Blake and Eric Liebler are employees of electroCore, Inc., and receive stock ownership. Georgios Giannakopoulos owns stock in electroCore, Inc. In the previous three years, Robert Levy has served as a consultant for Abbott, Saluda, Nalu, and Mainstay Medical and has stock options with Nalu and Saluda.
\end{abstract}

\section{INTRODUCTION}

The outbreak of coronavirus disease 2019 (COVID-19), caused by severe acute respiratory syndrome coronavirus 2 (SARS-CoV-2), which originated in Wuhan, China (1), has spread rapidly and was declared a global pandemic by the World Health Organization on March 11, 2020 (2). The clinical spectrum of COVID-19 broadly varies from asymptomatic to critical illness (3). As of April 21, 2020, more than 2.5 million confirmed cases and more than 175,000 deaths had been reported across 185 countries/regions (4). Patients who are most severely affected report moderate to severe respiratory symptoms (5), develop pneumonia and/or acute respiratory distress syndrome (ARDS), and require intensive care and/or respiratory support $(3,5,6)$. An extreme immune reaction, often referred to as a cytokine storm, that results in elevated levels of inflammatory cytokines including interleukin (IL)-6 appears to be linked to increased mortality from COVID-19 (7-9),
Address correspondence to: Eric Liebler, electroCore, Inc. 150 Allen Rd, Suite 201, Basking Ridge, NJ 07920, USA. Email: eric.liebler@electrocore.com

\footnotetext{
* electroCore, Inc., Basking Ridge, NJ, USA

${ }^{\dagger}$ Bayshore Medical Center, Hackensack Meridian Health, Holmdel, NJ, USA; and * Anesthesia Pain Care Consultants, Tamarac, FL, USA
}

For more information on author guidelines, an explanation of our peer review process, and conflict of interest informed consent policies, please go to http:// www.wiley.com/WileyCDA/Section/id-301854.html

Source(s) of Financial Support: Writing assistance and editorial support were provided by Elizabeth S. O. Barton, MS, of MedLogix Communications, LLC, in cooperation with the authors and were funded by electroCore, Inc.

This is an open access article under the terms of the Creative Commons Attribution-NonCommercial-NoDerivs License, which permits use and distribution in any medium, provided the original work is properly cited, the use is non-commercial and no modifications or adaptations are made.

www.neuromodulationjournal.com @ 2020 electroCore, Inc. Neuromodulation: Technology at the Neural Interface Neuromodulation 2020; 23: 784-788 published by Wiley Periodicals LLC. on behalf of International Neuromodulation Society. 


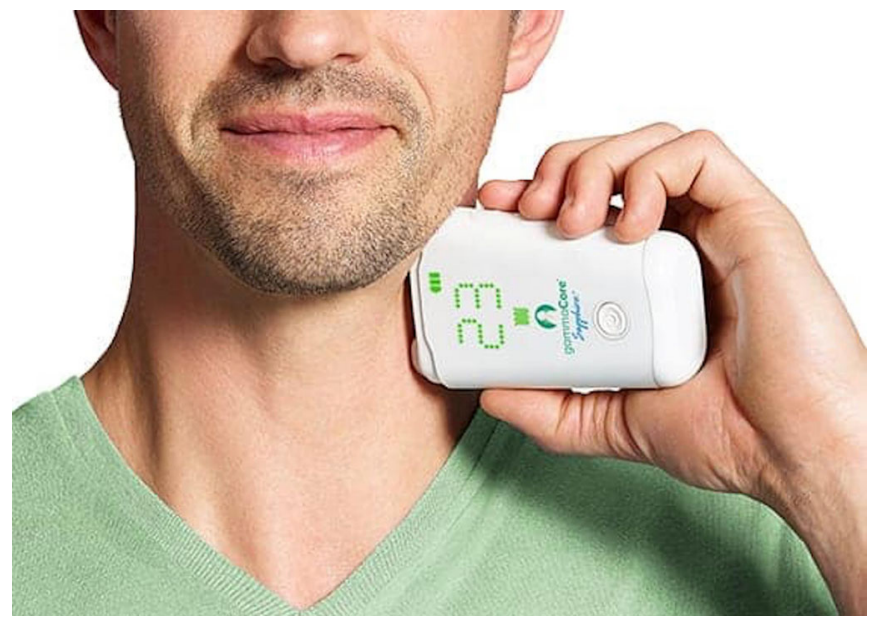

Figure 1 Treatment with non-invasive vagus nerve stimulation. [Color figure can be viewed at wileyonlinelibrary.com]

and strategies to mitigate the cytokine storm are being considered at this time. Symptomatic control has been complicated by concerns about inadvertent viral spread associated with the use of nebulizers and steroids, both of which are mainstays of conventional treatment for bronchoconstriction.

Vagus nerve stimulation (VNS) has been shown to be a potent modulator of pathologic immune reactions, specifically suppressing inflammatory cytokine levels via activation of the cholinergic anti-inflammatory pathway (CAP) (10-12). Inflammatory cytokines have been modulated by VNS in a variety of acute and progressive inflammatory conditions ranging from septic shock $(13,14)$ and asthma (15) to stroke $(16)$ and rheumatoid arthritis (17). In models of acute septic shock, VNS consistently demonstrated life-saving potential by reducing the expression of inflammatory cytokines (13).

Non-invasive vagus nerve stimulation (nVNS; gammaCore ${ }^{\mathrm{TM}}$, electroCore, Inc.; Fig. 1) is a neuromodulatory therapy that is U.S. Food and Drug Administration approved for acute and preventive treatment of migraine and cluster headache. It is CE marked in the EU for the treatment and prevention of symptoms of reactive airway disease, which includes asthma, bronchoconstriction, exercise-induced bronchospasm, and chronic obstructive pulmonary disease (COPD), as well as for a number of other conditions. Clinical data suggest that nVNS might provide benefits in patients who have respiratory symptoms that are sometimes associated with COVID-19, such as acute bronchoconstriction due to asthma (18) and respiratory distress associated with COPD (19).

Mechanistic and clinical data also demonstrate the potential for nVNS to mitigate the elevated cytokine levels caused by the cytokine storm in patients with COVID-19 $(20,21)$. The excellent safety and tolerability profile of nVNS further strengthens the case for its study and use in patients with COVID-19. We now share our early clinical observations from two patients treated with nVNS for the treatment of respiratory symptoms associated with COVID-19.

\section{MATERIALS AND METHODS}

Information was obtained via email correspondence and patient phone interviews. Informed consent was obtained for the publication of the patients' case reports.

\section{RESULTS}

\section{Case 1}

On March 23, 2020, a 50-year-old man developed symptoms of severe body aches; painful, unrelenting cough; a tingling sensation along the midline of his chest; anosmia; ageusia; and dyspnea. He was admitted to New York-Presbyterian Lawrence Hospital in Bronxville, New York, in the early morning of March 24. The patient's intercurrent medical conditions included type 2 diabetes mellitus, hypertension, sleep apnea, and seasonal allergies. He also had a history of hypercholesterolemia and ongoing use of a continuous positive airway pressure device for sleep apnea for 15 years. There was no history of smoking. Ongoing medications at hospital admission included ramipril $(10 \mathrm{mg} / \mathrm{d}$, for hypertension), metformin (2000 mg/d, for diabetes), glipizide (10 mg/d, for diabetes), and atorvastatin $(40 \mathrm{mg} / \mathrm{d}$ for hypercholesterolemia). At that time, the patient was tachycardic and hypertensive, and his blood glucose level was elevated. He was afebrile; the highest recorded body temperature was $99.0^{\circ} \mathrm{F}$. Chest X-ray, electrocardiogram, and laboratory results were normal, and myocardial infarction was ruled out. He was provided supplemental oxygen ( $2 \mathrm{~L}$ via nasal cannula) but reported that he repeatedly stopped using it, owing to nasal dryness and epistaxis. His oxygen saturation was monitored (lowest recorded measurement, 93\%). He received oral oxycodone $(5 \mathrm{mg}) /$ acetaminophen $(325 \mathrm{mg}$ ) every four hours for pain. For cough, he initially received dextromethorphan/guaifenesin/acetaminophen every four hours, which was subsequently replaced with hydrocodone bitartrate/ homatropine methyl bromide ( $5 \mathrm{~mL}$ every six hours).

On March 25, nasopharyngeal swab testing confirmed the presence of SARS-CoV-2. All other viral swab cultures were negative. He was treated with oral hydroxychloroquine $(600 \mathrm{mg})$, oral azithromycin ( $500 \mathrm{mg}$ for one day followed by $250 \mathrm{mg} / \mathrm{d}$ for four days), and inhaled albuterol as needed. A nebulizer was not used owing to concerns about potentially aerosolizing SARS-CoV-2. He continued to have severe, unrelenting cough and body aches, but his dyspnea had subsided, except during coughing bouts. He was discharged from the hospital and returned home on March 26. At the time of discharge, his blood glucose level and blood pressure were within normal levels, and his heart rate, although still elevated, had decreased to $\sim 90$ beats/min. His main symptoms of cough and body aches were adequately controlled with medication.

The patient, a clinician who had familiarity with neuromodulation through his practice, began using nVNS to treat his respiratory symptoms and expedite recovery at home. His typical treatment regimen consisted of two consecutive 120-second periods of stimulation (one on each side of the neck) administered approximately every three hours while awake. He reported using additional nVNS treatment (two stimulation episodes) as needed, approximately once per day. He discontinued use of both oxycodone/acetaminophen and hydrocodone bitartrate/ homatropine methyl bromide on March 28, two days after hospital discharge. On April 2, with continued use of nVNS every three hours when awake, his main symptoms were fatigue and low appetite, and coughing bouts had become rare. At last communication on April 7, he reported that he continued to use nVNS three times per day.

\section{Case 2}

On March 15, 2020, a 49-year-old man, a PhD scientist, with a 40-year history of asthma and no history of smoking experienced 
excessive fatigue. The following day, he developed symptoms of fever (highest recorded oral temperature, $100.6^{\circ} \mathrm{F}$ ), chills, cough, throat soreness, mental cloudiness, and chest tightness. He noted that his cough was usually dry, but was sometimes productive, and he periodically used airway clearance exercises, which he had previously used for his asthma. On March 18, he phoned his physician, who advised testing for COVID-19. The test was performed via nasopharyngeal swab at Kaiser Permanente South Baltimore County Medical Center on March 19 and processed by Quest Diagnostics $^{\mathrm{TM}}$. Test results, available on March 27, confirmed the presence of SARS-CoV-2.

At the time his COVID-19 symptoms began, the patient was taking pantoprazole $(80 \mathrm{mg} / \mathrm{d})$ for gastroesophageal reflux disease, loratadine $(20 \mathrm{~m} / \mathrm{d})$ for allergies, and montelukast $(10 \mathrm{mg} / \mathrm{d})$ for asthma. Previously, he had used nVNS, with or without inhaled levalbuterol to prevent wheezing related to his asthma, but he had not used either treatment during the preceding approximately six weeks.

On March 17, he began using nVNS therapy (one 120-second period of stimulation on the right side of the neck, two to three times per day) for COVID-19-associated symptoms of dyspnea and chest pressure/tightness. nVNS use was reported to provide immediate and consistent symptom relief and an improvement in his ability to clear his lungs. By March 21, the patient's fever, body aches, and mental cloudiness had ceased. On March 22, his asthma symptoms worsened, and he resumed use of inhaled levalbuterol concomitantly with nVNS therapy, as needed. He was prescribed an antibiotic (azithromycin) and oral steroids on March 23 but indicated that he did not take the oral steroids as he continued to experience substantial relief from his respiratory symptoms with use of nVNS and levalbuterol. He was also worried about sustained shedding of the virus due to immunosuppression with the steroids (22). As of April 2, his remaining symptom was a periodic, unproductive cough.

\section{DISCUSSION}

These cases highlight the potential utility of nVNS in COVID-19 symptom management. In both cases, nVNS therapy rapidly provided clinically relevant benefits to the patients. In case 1 , the patient used nVNS to aid in his recovery at home after hospital discharge. He was able to discontinue use of opioid and cough suppressant medications with nVNS use. In case 2, the patient experienced immediate (within five minutes of the stimulation) and reliable relief from chest tightness and dyspnea, as well as an improved ability to clear his lungs when he used nVNS to manage his symptoms at home. Avoidance of ventilator dependence in both cases and lack of hospitalization in case 2 cannot be causally attributed to nVNS, but it is reasonable to speculate that nVNS therapy may have been a supporting factor, via either bronchodilation or inhibition of cytokine release. In light of significant concerns about inadequate numbers of intensive care unit beds and ventilators amid the rapid spread of COVID-19, it is imperative to consider all viable therapeutic options that could lead to symptomatic improvement and decrease the need for hospitalization and subsequent mechanical ventilation. The well-established safety and tolerability of nVNS therapy make it a particularly attractive treatment modality that merits immediate study.

Information about the mechanisms and the clinical spectrum of COVID-19 is developing rapidly. Data thus far suggest that the viral infection is capable of producing an extreme immune reaction in the host, often referred to as a cytokine storm $(8,9)$. IL-6, which is produced by activated leukocytes and acts on several cell and tissue types, is believed to be a key cytokine in COVID-19; IL-6 levels have been closely linked with disease severity and progression (7,8). Clinically, respiratory symptoms of COVID-19 appear to stem from inflammatory-induced lung injury (8) and cause a decrease in oxygen saturation ( $\leq 93 \%$ in severe cases) (5). This leads to rapid deterioration of respiratory function, which, if not mitigated, can result in ARDS, diffuse organ involvement, and, in many cases, death $(1,23,24)$. Therapies that mitigate or block the cytokine storm could potentially decrease the need for long-term respiratory support and mechanical ventilation. Ultimately, this could lead to decreased mortality.

There are two presumed mechanisms by which VNS may help patients with respiratory distress due to COVID-19. First, the smooth muscles found in the walls of bronchi and bronchioles regulate the flow of air into the lungs (25). Under normal conditions, when greater volumes of air are required by the body, smooth muscle relaxes to dilate the bronchi and bronchioles (26). When the inflammatory response is dysregulated, bronchoconstriction results, limiting the flow of air to and from the lungs. VNS may inhibit this airway constriction through a parasympathetic-sympathetic reflex arc, whereby stimulation of an afferent vagal nerve causes an efferent sympathetically mediated release of catecholamines that results in smooth muscle relaxation (27). This represents an additional benefit of VNS, considering the potential for viruses to induce asthmatic reactions (28).

The potential for nVNS to decrease cough may be of particular benefit for patients with COVID-19, as mitigating cough at any stage of the disease is likely to curb transmission. For patients with asthma caused by viruses such as COVID-19 or other respiratory conditions, nebulizers are often prescribed acutely to deliver corticosteroids and/or other therapeutic agents. As illustrated in case 2, nVNS may represent an alternative therapy for airway reactivity. This could potentially decrease the use of both nebulizers and corticosteroids in patients with COVID-19, which has been recommended by the World Health Organization owing to concerns about furthering the spread of SARS-CoV-2 (29,30). Animal and human models have demonstrated a catecholamine mechanism of action and clinical utility of VNS in the emergency department for patients with severe airway reactivity. Moreover, with growing concern that nebulizers or steroids could increase viral spread, it is desirable to attain symptomatic control without this risk.

Second, the CAP is believed to be the vagus nerve-based arm of the inflammatory reflex (31). Evidence indicates that stimulation of the CAP leads to acetylcholine (ACh) binding to $\alpha-7$-nicotinic ACh receptors ( $\alpha 7 n A C h R s)$, resulting in reduced production of the inflammatory cytokines tumor necrosis factor (TNF), IL-1 $\beta$, and IL-6 (31-33). The use of the CAP to regulate the inflammatory response has been investigated broadly using varying forms of VNS for more than 20 years $(10,32)$. The use of nVNS to target neural pathways to treat inflammatory diseases represents the most recent exploration of this pathway.

Clinical studies that support the utility of nVNS to affect both of these mechanistic pathways are listed and summarized in Table 1. Preliminary evidence from multiple clinical trials suggests that nVNS could ease the breathing and confer symptomatic relief in patients with asthma or COPD $(18,19,34)$. Initial human clinical work was performed with a minimally invasive form of VNS delivered through a percutaneous electrode in severely compromised patients with asthma who had a forced expiratory volume in one second $\left(\mathrm{FEV}_{1}\right)$ that was $25 \%-75 \%$ of normal (35). A 
Table 1 Summary of nVNS Trials Relevant to COVID-19.

\begin{tabular}{|c|c|c|c|}
\hline Study & Design & $N$ & Findings relevant to COVID-19 \\
\hline \multicolumn{4}{|l|}{ Respiratory clinical trials } \\
\hline $\begin{array}{l}\text { nVNS for the treatment of } \\
\text { acute asthma } \\
\text { exacerbations (18) }\end{array}$ & $\begin{array}{l}\text { Prospective, multicenter, } \\
\text { open-label study }\end{array}$ & 4 & $\begin{array}{l}\text { Ninety minutes after acute } \mathrm{nVNS} \text { treatment, } \mathrm{FEV}_{1} \text { improved from } \\
\text { baseline by a mean of } 73 \% \text {, and mean VAS dyspnea score } \\
\text { decreased from } 8 \text { (at baseline) to } 1\end{array}$ \\
\hline $\begin{array}{l}\text { nVNS for the relief of acute } \\
\text { bronchoconstriction due } \\
\text { to asthma }(25,34)\end{array}$ & $\begin{array}{l}\text { Prospective, multicenter, } \\
\text { open-label study }\end{array}$ & 30 & $\begin{array}{l}\text { Ninety minutes after acute nVNS treatment, } 93 \% \text { of patients } \\
\text { reported improvement in VAS dyspnea score, and } 86 \% \text { had } \\
\text { improvements in FEV }\end{array}$ \\
\hline $\begin{array}{l}\text { nVNS for the prophylactic } \\
\text { treatment of COPD (19) }\end{array}$ & $\begin{array}{l}\text { Prospective, single-center, } \\
\text { randomized, controlled trial }\end{array}$ & 54 & $\begin{array}{l}\text { After } 8 \text { weeks of daily treatment, the nVNS group had a } \\
\text { significant increase from baseline in distance walked (9.9-m } \\
\text { greater improvement than the sham group) on the } 6 \mathrm{MWT}\end{array}$ \\
\hline \multicolumn{4}{|c|}{ Inflammatory clinical and mechanistic trials } \\
\hline $\begin{array}{l}\text { nVNS decreases whole } \\
\text { blood culture-derived } \\
\text { cytokines and } \\
\text { chemokines (20) }\end{array}$ & $\begin{array}{l}\text { Randomized, blinded, pilot trial in } \\
\text { healthy controls }\end{array}$ & 20 & $\begin{array}{l}\text { Twenty-four hours after treatment, the nVNS group had a greater } \\
\text { (vs the sham group) percentage decrease in levels of IL-1 } \beta \text {, } \\
\text { TNF, IL-6, IL-8, MIP-1 } \alpha \text {, and MCP-1 }\end{array}$ \\
\hline $\begin{array}{l}\text { Effects of nVNS on fatigue } \\
\text { and immune responses } \\
\text { in patients with primary } \\
\text { Sjögren's syndrome (21) }\end{array}$ & $\begin{array}{l}\text { Prospective, single-center, } \\
\text { open-label study }\end{array}$ & 15 & $\begin{array}{l}\text { Ninety minutes after } n V N \text {, levels of MIP- } 1 \alpha, I L-1 \beta, T N F-\alpha, I L-6 \text {, and } \\
\text { IP- } 10 \text { were significantly reduced on days } 0,7 \text {, and } 28 \text { of the } \\
\text { study } \\
\text { Twelve participants had an improvement in their fatigue scores, } \\
\text { seven of whom had a } \geq 30 \% \text { reduction in fatigue within } \\
28 \text { days }\end{array}$ \\
\hline
\end{tabular}

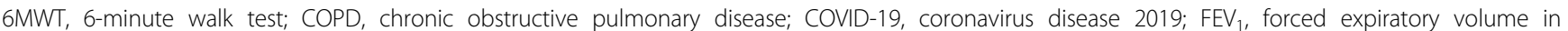
one second; IL, interleukin; IP, interferon $\gamma$-induced protein; MCP, monocyte chemoattractant protein; MIP, macrophage inflammatory protein; nVNS, non-invasive vagus nerve stimulation; TNF, tumor necrosis factor; VAS, visual analog scale.

continuous 60-minute stimulation of the vagus nerve at $25 \mathrm{~Hz}$ provided a clinically meaningful improvement (versus historical controls) in $\mathrm{FEV}_{1}$ and the work of breathing. In the second study, subjects received discrete 2-minute nVNS doses, and the change in $\mathrm{FEV}_{1}$ was measured 1,5 , and 90 minutes after stimulation $(25,34)$. Improvements in $\mathrm{FEV}_{1}$ were noted in 19 of the 29 subjects $(66 \%)$ at one minute, 18 subjects $(62 \%)$ at five minutes, and 25 subjects (86\%) at 90 minutes. Improvements in the work of breathing, peak expiratory flow, and visual analog scale of dyspnea scores were also reported.

Multiple studies also demonstrate the ability of nVNS to decrease the level of inflammatory cytokines. The first, conducted in healthy individuals, showed a greater percentage reduction in IL-1 $\beta, T N F$, IL-6, IL-8, macrophage inflammatory protein (MIP)-1 $\alpha$, and monocyte chemoattractant protein (MCP)-1 levels in nVNStreated subjects than in sham-treated control subjects (20). In patients with Sjögren's syndrome (an inflammatory immunemediated condition), levels of MIP- $1 \alpha$, IL-1 $\beta$, TNF- $\alpha$, IL- 6 , and interferon $\gamma$-induced protein (IP)-10 were significantly reduced 90 minutes after stimulation as well as at days 7 and 28 of the study (21). Recently, nVNS was found to downregulate levels of several inflammatory cytokines, including $\mathrm{IL}-6$, in patients with post-traumatic stress disorder (D. Bremner, MD, personal communication, April 14, 2020). In these studies, nVNS was used on a consistent basis, with additional acute use as needed for symptom control in some cases. This dosing regimen provides needed flexibility for patients whose respiratory symptoms may vary substantially over the course of their illness.

There is a strong scientific and clinical foundation for the hypothesis that nVNS can improve disease outcomes in patients with COVID19. Previous findings suggest that the clinical benefits from VNS and nVNS are driven by mechanisms of action relevant to COVID-19. The robust established safety and tolerability profile of nVNS strengthens the case for the additional investigation and use of nVNS to fight the
COVID-19 pandemic. An Emergency Use Authorization application was recently submitted to the U.S. Food and Drug Administration to facilitate use and further study of nVNS during the COVID-19 pandemic (36). Randomized controlled trials are being initiated at multiple sites in the United States and Europe.

\section{Acknowledgements}

The authors would like to acknowledge the significant contributions of J.P. Errico and Bruce Simon to the original development of nVNS and its subsequent clinical development.

\section{Authorship Statement}

All authors were involved in the collection and/or interpretation the data and contributed to the development of this report. The authors have approved the final version of the report and support the findings and conclusions stated herein.

\section{How to Cite this Article:}

Staats P., Giannakopoulos G., Blake J., Liebler E., Levy R.M. 2020. The Use of Non-invasive Vagus Nerve Stimulation to Treat Respiratory Symptoms Associated With COVID-19: A Theoretical Hypothesis and Early Clinical Experience. Neuromodulation 2020; 23: 784-788 


\section{REFERENCES}

1. Huang C, Wang Y, Li X et al. Clinical features of patients infected with 2019 nove coronavirus in Wuhan, China. Lancet 2020;395:497-506.

2. World Health Organization. WHO Director-General's opening remarks at the media briefing on COVID-19 -11 March 2020. https://www.who.int/dg/speeches/ detail/who-director-general-s-opening-remarks-at-the-media-briefing-on-covid19-11-march-2020

3. Goh KJ, Choong MC, Cheong EH et al. Rapid progression to acute respiratory distress syndrome: review of current understanding of critical illness from COVID-19 infection. Ann Acad Med Singapore 2020;49:108-118.

4. COVID-19 dashboard by the Center for Systems Science and Engineering (CSSE) at Johns Hopkins University (JHU). https://gisanddata.maps.arcgis.com/apps/ opsdashboard/index.html\#/bda7594740fd40299423467b48e9ecf6

5. Wu Z, McGoogan JM. Characteristics of and important lessons from the coronavirus disease 2019 (COVID-19) outbreak in China: summary of a report of 72314 cases from the Chinese Center for Disease Control and Prevention. JAMA 2020 323:1239-1242. https://doi.org/10.1001/jama.2020.2648

6. Jiang F, Deng L, Zhang L, Cai Y, Cheung CW, Xia Z. Review of the clinical charac teristics of coronavirus disease 2019 (COVID-19). J Gen Intern Med 2020; e-pub ahead of print. https://doi.org/10.1007/s11606-020-05762-w.

7. Ulhaq ZS, Soraya GV. Interleukin-6 as a potential biomarker of COVID-19 progression. Med Mal Infect 2020; e-pub ahead of print. https://doi.org/10.1016/j. medmal.2020.04.002

8. Zhang $\mathrm{C}, \mathrm{Wu} \mathrm{Z}$, Li JW, Zhao $\mathrm{H}$, Wang GQ. The cytokine release syndrome (CRS) of severe COVID-19 and interleukin-6 receptor (IL-6R) antagonist tocilizumab may be the key to reduce the mortality. Int J Antimicrob Agents 2020;105954. https://doi.org/10.1016/j.ijantimicag.2020.105954.

9. Zhou F, Yu T, Du R et al. Clinical course and risk factors for mortality of adult inpatients with COVID-19 in Wuhan, China: a retrospective cohort study. Lance 2020;395:1054-1062

10. Tracey KJ. The inflammatory reflex. Nature 2002;420:853-859.

11. Pavlov VA, Chavan SS, Tracey KJ. Bioelectronic medicine: from preclinical studies on the inflammatory reflex to new approaches in disease diagnosis and treatment. Cold Spring Harb Perspect Med 2020;10:a034140.

12. Tracey KJ. Physiology and immunology of the cholinergic antiinflammatory path way. J Clin Invest 2007;117:289-296.

13. Borovikova LV, Ivanova $S$, Zhang $M$ et al. Vagus nerve stimulation attenuates the systemic inflammatory response to endotoxin. Nature 2000;405:458-462.

14. Kessler W, Diedrich S, Menges P et al. The role of the vagus nerve: modulation of the inflammatory reaction in murine polymicrobial sepsis. Mediators Inflamm 2012;2012:467620.

15. Johnson RL, Murray ST, Camacho DK, Wilson CG. Vagal nerve stimulation attenuates IL- 6 and TNF $\alpha$ expression in respiratory regions of the developing rat brainstem. Respir Physiol Neurobiol 2016;229:1-4

16. Zhao M, He X, Bi XY, Yu XJ, Gil Wier W, Zang WJ. Vagal stimulation triggers peripheral vascular protection through the cholinergic anti-inflammatory pathway in a rat mode of myocardial ischemia/reperfusion. Basic Res Cardiol 2013;108:345

17. Koopman FA, Chavan SS, Miljko $S$ et al. Vagus nerve stimulation inhibits cytokine production and attenuates disease severity in rheumatoid arthritis. Proc Nat Acad Sci U S A 2016;113:8284-8289.

18. Steyn E, Mohamed Z, Husselman C. Non-invasive vagus nerve stimulation for the treatment of acute asthma exacerbations-results from an initial case series. Int $J$ Emerg Med 2013;6:7.

19. Study to compare (COPD) assessment test in COPD stage II and IV in a prophylactic treatment. ClinicalTrials.gov identifier: NCT01679314. https:// clinicaltrials.gov/ct2/show/results/NCT01679314?term=AC-001\&draw=2\& rank=2

20. Lerman I, Hauger $R$, Sorkin $L$ et al. Noninvasive transcutaneous vagus nerve stimulation decreases whole blood culture-derived cytokines and chemokines: a randomized, blinded, healthy control pilot trial. Neuromodulation 2016;19: 283-290.
21. Tarn J, Legg S, Mitchell S, Simon B, Ng WF. The effects of noninvasive vagus nerve stimulation on fatigue and immune responses in patients with primary Sjögren's syndrome. Neuromodulation. 2019;22:580-585.

22. Cao B, Gao H, Zhou B et al. Adjuvant corticosteroid treatment in adults with influenza a (H7N9) viral pneumonia. Crit Care Med 2016;44:e318-e328.

23. Ahmadpoor $P$, Rostaing $L$. Why the immune system fails to mount an adaptive immune response to a Covid-19 infection. Transpl Int 2020; e-pub ahead of print. https://doi.org/10.1111/tri.13611.

24. Pedersen SF, Ho YC. SARS-CoV-2: a storm is raging. J Clin Invest 2020; e-pub ahead of print. https://doi.org/10.1172/JCI137647.

25. Staats $P$, Emala C, Simon B, Errico JP. Neurostimulation for asthma. In: Krames ES Peckham PH, Rezai AR, editors. Neuromodulation: comprehensive textbook of prin ciples, technologies, and therapies. Volume 1. 2nd ed. London, UK: Elsevier, 2018: 1339-1345.

26. White SR, Sands MF, Popovich KJ et al. Homeostatic regulation of airway smooth muscle tone by catecholamine secretion in swine. J Appl Physiol (1985) 1987;62: 972-977.

27. Hoffmann TJ, Mendez S, Staats P, Emala CW, Guo P. Inhibition of histamineinduced bronchoconstriction in guinea pig and swine by pulsed electrical vagus nerve stimulation. Neuromodulation. 2009;12:261-269.

28. Jacoby DB. Virus-induced asthma attacks. J Aerosol Med 2004;17:169-173.

29. World Health Organization. Modes of transmission of virus causing COVID-19: implications for IPC precaution recommendations. https://www.who.int/news$\mathrm{room} /$ commentaries/detail/modes-of-transmission-of-virus-causing-covid-19implications-for-ipc-precaution-recommendations

30. World Health Organization. Clinical management of severe acute respiratory infection (SARI) when COVID-19 disease is suspected. https://www.who.int/docs/ default-source/coronaviruse/clinical-management-of-novel-cov.pdf?sfvrsn= bc7da517_2

31. Pavlov VA, Tracey KJ. The vagus nerve and the inflammatory reflex--linking immunity and metabolism. Nat Rev Endocrinol 2012;8:743-754.

32. Andersson U, Tracey KJ. Reflex principles of immunological homeostasis. Annu Rev Immunol 2012;30:313-335.

33. Kaczmarczyk R, Tejera D, Simon BJ, Heneka MT. Microglia modulation through external vagus nerve stimulation in a murine model of Alzheimer's disease. J Neurochem 2017;146:76-85.

34. Relief of acute bronchoconstriction/asthma using the non-invasive AlphaCore device. ClinicalTrials.gov identifier: NCT01532817. https:// clinicaltrials.gov/ct2/show/results/NCT01532817?term=alphacore\&cond= Asthma\&draw $=2 \&$ rank $=1$

35. Miner JR, Lewis LM, Mosnaim GS, Varon J, Theodoro D, Hoffmann TJ. Feasibility of percutaneous vagus nerve stimulation for the treatment of acute asthma exacerbations. Acad Emerg Med 2012;19:421-429.

36. electroCore submits Emergency Use Authorization application to allow the study and use of gammaCore nVNS therapy to treat respiratory symptoms associated with COVID-19. https://apnews.com/Globe\%20Newswire/112444f0a1cb14809a58 $0 c 36 b b 73428 b$

\section{COMMENT}

This article is timely and extremely important and should be published without hesitation. VNS might just be the right treatment for severe symptoms due to the cytokine storm of Covid-19.

Elliot Krames, MD San Francisco, CA USA

Comments not included in the Early View version of this paper 